\section{ORIGINAL RESEARCH}
H.-M. Yoo
S.J. Kim
C.G. Choi
D.H. Lee
J.H. Lee
D.C. Suh
J.W. Choi
K.S. Jeong
S.J. Chung
J.S. Kim
S.-C. Yun

\title{
Detection of CSF Leak in Spinal CSF Leak Syndrome Using MR Myelography: Correlation with Radioisotope Cisternography
}

\begin{abstract}
BACKGROUND AND PURPOSE: Spinal CSF leak syndrome is a unique disorder caused by spinal CSF leak. In this study, we attempted to determine whether MR myelography (MRM) can detect the leakage site in the spine.

MATERIALS AND METHODS: We performed both MRM and radioisotope cisternography (RIC) in 15 patients with spinal CSF leak syndrome. Patients were included in this study if they had at least 2 of the following criteria: 1) orthostatic headache, 2) low CSF opening pressure, and 3) diffuse pachymeningeal enhancement on brain MR imaging. For comparison, we performed MRM in 15 subjects without symptoms of spinal CSF leak syndrome. MRM was performed with the 2D turbo spin-echo technique in the entire spine by using a 1.5T scanner. Two blinded radiologists evaluated the MRM findings in a total of 30 cases, composed of patient and control groups, with regard to the presence of leakage and the level of leakage if present. RIC was performed only in the patient group and was assessed by consensus among 3 physicians experienced in nuclear medicine. The diagnostic performance of MRM and RIC was evaluated on the basis of the clinical diagnosis of spinal CSF leak syndrome.
\end{abstract}

RESULTS: The sensitivity, specificity, and accuracy of MR myelography for detecting CSF leak was $86.7 \%, 86.7 \%$, and $86.7 \%$ for reader 1 , respectively, and $80.0 \%, 93.3 \%$, and $86.7 \%$ for reader 2 , respectively. The sensitivity of RIC was 93.3\%. Agreement between the 2 techniques for the detection of CSF leak was substantial in reader 1 and moderate in reader 2 ( $\kappa=0.634$ and 0.444 , respectively).

CONCLUSION: MRM is an effective tool for detecting CSF leak in the spine in patients with spinal CSF leak syndrome.

$S^{\mathrm{p}}$ pinal CSF leak syndrome is a rare disorder characterized by postural headache and low CSF pressure $(<60 \mathrm{~mm}$ $\mathrm{H}_{2} \mathrm{O}$ ). It is currently accepted that the major causative factor of spinal CSF leak syndrome is the release of CSF in the spinal column. ${ }^{1}$

The diagnosis of spinal CSF leak syndrome may be challenging because various manifestations of this syndrome can be encountered. ${ }^{2,3}$ The syndrome is generally considered to be benign, and most patients improve spontaneously or with conservative management. Treatment options include analgesics, sedatives, antiemetics, intravenous hydration, epidural blood patch (EBP) or epidural saline infusion, and systemic steroids. Among these treatment modalities, autologous EBP is considered the treatment of choice for those patients who have failed the initial conservative treatment. ${ }^{4,5}$ Detection of the leakage site is important for the use of a targeted EBP because placing this patch at the level of the leak is generally considered more effective than placing it at a distant site and it usually provides long-term relief. ${ }^{4,5}$

Localization of a CSF leak can be attempted by invasive diagnostic techniques such as radioisotope cisternography

\section{Received September 6, 2007; accepted after revision October 27.}

From the Departments of Radiology (H.-M.Y., S.J.K., C.G.C., D.H.L., J.H.L., D.C.S., J.W.C., K.S.J.), Neurology (S.J.C.), Nuclear Medicine (J.S.K.), and Preventive Medicine (S.-C.Y.), Asan Medical Center, University of Ulsan College of Medicine, Seoul, South Korea; and Department of Radiology (H.M.Y.), Konyang University College of Medicine, Chungnam, South Korea

Please address correspondence to Sang Joon Kim, MD, Asan Medical Center, University of Ulsan College of Medicine, 388-one Pungnap 2-dong; Songpa-gu, Seoul, 138-736, Korea; e-mail: sjkimjb@amc.seoul.kr

DOI 10.3174/ajnr.A0920
(RIC) or CT cisternography (CTC). MR myelography (MRM) is a noninvasive method that can also be used for demonstrating CSF leak. It has no radiation hazard and can be performed without intrathecal administration of contrast media or radioisotopes, unlike CTC or RIC. There have been sporadic case reports in which MRM was used for the detection of a CSF leak. ${ }^{6}$ We tested to see if MRM could detect the leakage site in 15 consecutive patients with spinal CSF leak syndrome and compared the results of MRM with those of RIC. For comparison, we performed MRM in 15 subjects without symptoms of spinal CSF leak syndrome.

\section{Materials and Methods}

We studied 15 patients with spinal CSF leak syndrome. Patient inclusion in this study depended on their fulfilling at least 2 of the following 3 criteria: 1) orthostatic headache, 2) low CSF pressure, and 3) diffuse pachymeningeal enhancement on brain MR imaging. ${ }^{1}$

The average patient was 37.6 years of age (range, 17-53 years). There were 5 men and 10 women. Both MRM and RIC were performed on all patients. The mean interval between the MRM and RIC was 2 days (range, 1-5 days). MRM was performed before RIC in 7 patients and after RIC in 8 patients. Follow-up MRM was performed in 6 of the 13 patients who were treated with EBP.

RIC was performed following lumbar injection of $0.4 \mathrm{~mL}$ of technetium Tc99m diethylenetriamine penta-acetic acid containing 5-7 mCi. Images were obtained 30 minutes and 2, 4, and 6 hours after injection.

MRM was performed by using a 2D turbo spin-echo (TSE) technique in the entire spine level on a 1.5T MR imaging scanner (Gyroscan Intera; Philips, Eindhoven, the Netherlands). The entire spine 
was scanned in 2 separate levels (ie, the cervicothoracic area and the thoracolumbar area). The scanning parameters were as follows: TR, $8000 \mathrm{~ms}$; TE, $1000 \mathrm{~ms}$; FOV, $270 \mathrm{~mm}$; matrix size, $512 \times 512$; section thickness, $50 \mathrm{~mm}$; section orientation, coronal; and TSE factor, 256. The acquisition time for each level was 72 seconds.

For comparison with the patient group, MRM was performed in 15 subjects who underwent spinal MR imaging to evaluate low-back pain or other symptoms but did not have symptoms of spinal CSF leak syndrome. The mean age of the control group was 37.3 years (range, $18-59$ years), and the ratio between men and women was 2:1. RIC was not performed in this control group. Written permission was obtained for MRM or RIC in all patients or members of the control group.

We evaluated the MRM and RIC with regard to the presence of leakage and the level of leakage if present. Two radiologists (J.W.C., K.S.J.), who were blinded to the clinical information, independently assessed the MRM. One of the authors (S.J.K.) made criteria for CSF leak on MRM after reviewing MRM and RIC findings of the patient and control groups. CSF leak was graded with a 4-point scale: Grade zero refers to absence of CSF leak; grade 1 refers to possible leakage showing a triangular-shaped expansion of the CSF space column around the nerve root sleeves; grade 2 refers to probable leakage showing high signal intensity lateral to the nerve root sleeves, in addition to triangular expansion around the nerve root sleeve but with the length less than the width of the thecal sac; and grade 3 refers to definite CSF leak showing profuse extraspinal high signal intensity lateral to and around the nerve root sleeves with the length more than the width of the thecal sac (Fig 1). Leakage was assessed at each level of the spine separately (ie, cervical, thoracic, and lumbar level); the cervicothoracic junction was included to the thoracic level and the thoracolumbar junction, to the lumbar level. MRM was considered positive for leakage when the grade was 1 or higher at any 1 level or more. Evaluation was performed on our PACS with a window width setting of 1800-2000 and a window level setting of -600 to -800 to obtain high-contrast images.

RIC was evaluated by the consensus of 3 physicians who were experienced in nuclear medicine. CSF leak was assessed at each level of the spine with a grading system similar to that of MRM from grade zero for no leakage (no paraspinal activity) to grade 3 for definite leakage (hot paraspinal activity with the length over the transverse diameter of spinal canal activity) (Fig 1). RIC was considered positive when there was grade 1 or higher leakage of CSF at any 1 level or more.

The sensitivity, specificity, and accuracy of MRM were assessed on the basis of the clinical diagnosis of spinal CSF leak syndrome, which we used as the reference standard. RIC was performed only in the patient group, so the evaluation was not blinded and only the sensitivity of the RIC was calculated. Interobserver agreement of the evaluation results of MRM between the 2 radiologists was analyzed by using $\kappa$ statistics. Agreement between MRM and RIC findings was also analyzed. The Statistical Package for the Social Sciences for Windows (Version 12.0; SPSS, Chicago, Ill) was used for statistical analyses.

\section{Results}

Reader 1 detected 13 cases of CSF leak on MRM among 15 of the disease group, whereas reader 2 detected 12 (Fig 2). On RIC, 14 cases of CSF leak were detected. The results are summarized in Table 1. Among 15 subjects of the control group, reader 1 reported grade 1 leakage in 2 subjects and reader 2 , in 1. In the remaining subjects, they reported no leakage. The

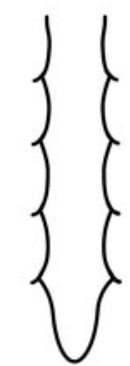

Grade 0

A

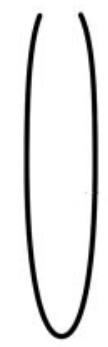

Grade 0

B

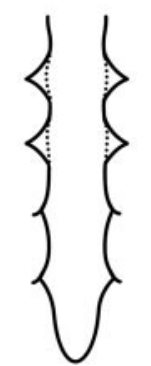

Grade 1

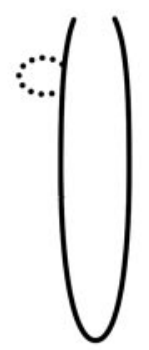

Grade 1

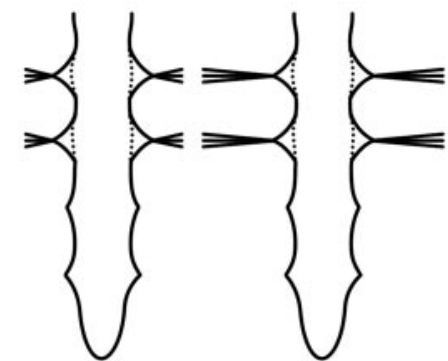

Grade 2

Grade 3

Grade 2

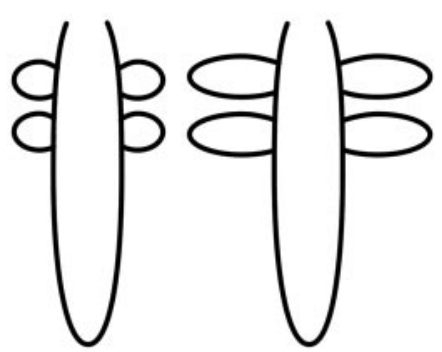

Grade 3
Fig 1. Schematic drawing of 4-point-scale grading system of CSF leak on MRM $(A)$ and RIC (B). A, Our grading scale on MRM is depicted as follows: grade zero, no leak (normal findings on MR myelogram); grade 1, possible leak (expansion of the CSF space column around the nerve root sleeve); grade 2, probable leak (streaky hyperintensity lateral to the nerve root sleeves but with length less than the transverse diameter of the thecal sac); and grade 3, definite leak (lateral extension greater than the transverse diameter of the thecal sac). However, actual grading of the case is determined on the basis of the highest grade in each level of the spine. B, Grade of CSF leak on RIC is depicted as follows: grade zero, no paraspinal activity; grade 1, possible leak (faint paraspinal activity with length under the transverse diameter of spinal canal activity); grade 2, probable leak (hot paraspinal activity with the length under the transverse diameter of spinal canal activity); and grade 3, definite leak (hot paraspinal activity with the length over the transverse diameter of spinal canal activity)

sensitivity, specificity, and accuracy of MRM for detecting CSF leak were $86.7 \%, 86.7 \%$, and $86.7 \%$ for reader 1 , respectively, and $80.0 \%, 93.3 \%$, and $86.7 \%$ for reader 2 , respectively. The sensitivity of RIC was $93.3 \%$ (Table 2 ). Specificity and accuracy were not calculated in RIC because RIC was not performed in the control group. Agreement between the 2 techniques for detection of CSF leak was substantial in reader 1 and moderate in reader $2(\kappa=0.634$ and 0.444 , respectively). Agreement between the 2 readers for detection of leakage on MRM was almost perfect $(\kappa=0.867)$

The leakage site seen on MRM and treatment for the patients with CSF leak are summarized in Table 1. On MRM, the thoracic spine, in which we included the cervicothoracic junction, was the most frequent location of CSF leak (reader $1, n=$ 7; reader $2, n=8$; and RIC, $n=11$ ) followed by the lumbar spine (reader $1, n=6$; reader $2, n=7$; and RIC, $n=4$ ). All patients who were considered to have CSF leak showed multiple sites of CSF leakage.

Two of the 15 patients received only supportive measures, such as bed rest and intravenous hydration, with symptomatic improvement. Thirteen of the 15 patients were treated by using an EBP based on the leakage site seen on MRM and RIC. In 7 patients, repeated EBPs were used due to persistent headache. All 13 patients improved clinically after a single or re- 

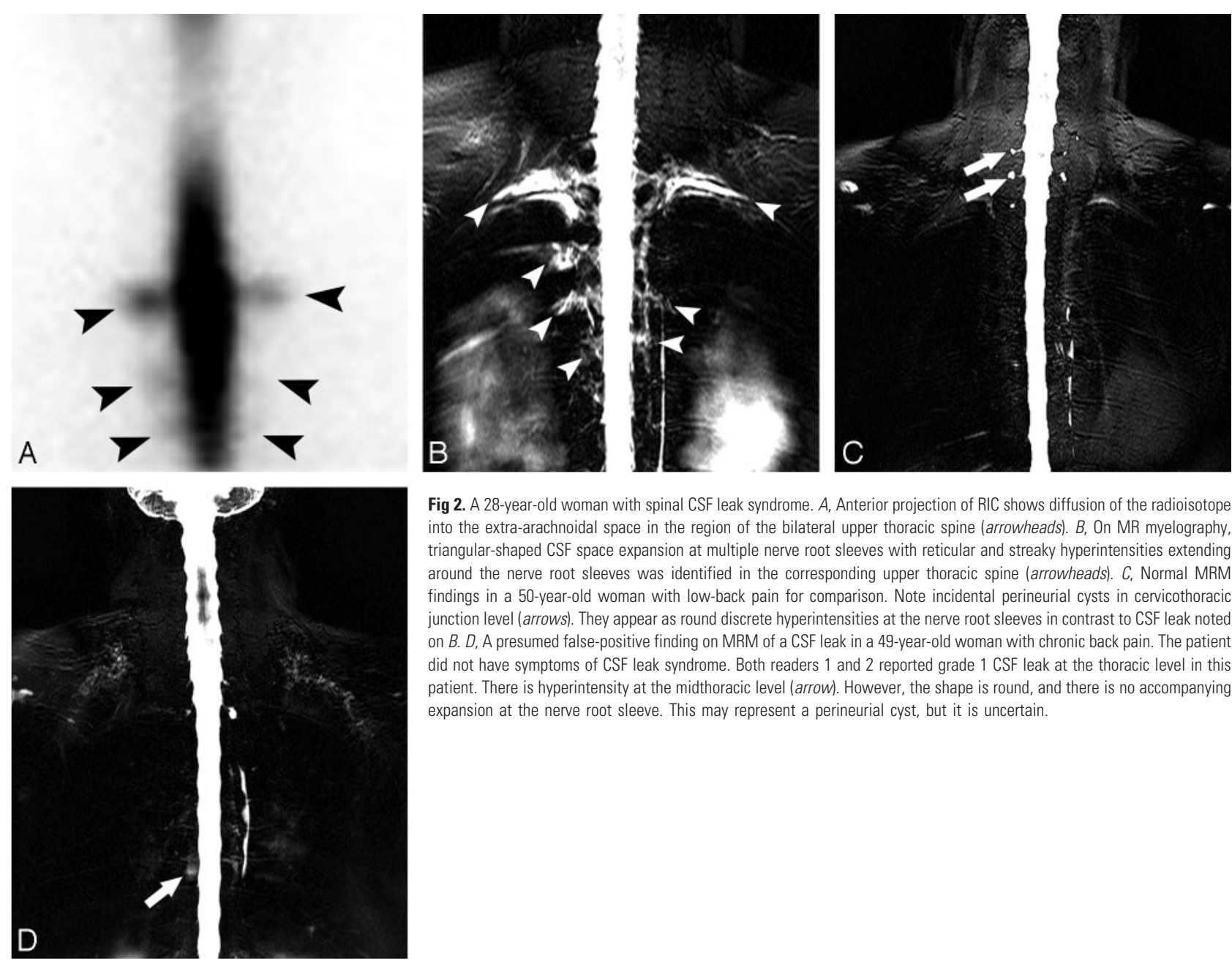

Fig 2. A 28-year-old woman with spinal CSF leak syndrome. A, Anterior projection of RIC shows diffusion of the radioisotope into the extra-arachnoidal space in the region of the bilateral upper thoracic spine (arrowheads). B, On MR myelography, triangular-shaped CSF space expansion at multiple nerve root sleeves with reticular and streaky hyperintensities extending around the nerve root sleeves was identified in the corresponding upper thoracic spine (arrowheads). C, Normal MRM findings in a 50-year-old woman with low-back pain for comparison. Note incidental perineurial cysts in cervicothoracic junction level (arrows). They appear as round discrete hyperintensities at the nerve root sleeves in contrast to CSF leak noted on $B . D$, A presumed false-positive finding on MRM of a CSF leak in a 49-year-old woman with chronic back pain. The patient did not have symptoms of CSF leak syndrome. Both readers 1 and 2 reported grade 1 CSF leak at the thoracic level in this patient. There is hyperintensity at the midthoracic level (arrow). However, the shape is round, and there is no accompanying expansion at the nerve root sleeve. This may represent a perineurial cyst, but it is uncertain.

\begin{tabular}{|c|c|c|c|c|c|}
\hline \multirow{3}{*}{$\begin{array}{l}\text { Patient } \\
\text { No. }\end{array}$} & \multirow{3}{*}{$\begin{array}{c}\text { Age } \\
(\mathrm{yr}) / \mathrm{Sex}\end{array}$} & \multicolumn{3}{|c|}{ Location of the Leak } & \multirow{3}{*}{$\begin{array}{r}\text { Treatment } \\
\text { (frequency) }\end{array}$} \\
\hline & & \multicolumn{2}{|c|}{ MRM } & \multirow[b]{2}{*}{ RIC } & \\
\hline & & Reader 1 & Reader 2 & & \\
\hline 1 & $42 / F$ & $\mathrm{~T} / \mathrm{L}$ & No & $T$ & EBP (3) \\
\hline 2 & $33 / \mathrm{M}$ & $\mathrm{C} / \mathrm{T}$ & $\mathrm{T} / \mathrm{L}$ & $\mathrm{T}$ & $\mathrm{EBP}(4)$ \\
\hline 3 & $52 / F$ & $\mathrm{No}^{*}$ & No* & $\mathrm{T}$ & $\mathrm{EBP}(1)$ \\
\hline 4 & $53 / F$ & $\mathrm{~L}$ & $\mathrm{~L}$ & $\mathrm{~L}$ & $\mathrm{EBP}(1)$ \\
\hline 5 & $45 / M$ & C & $\mathrm{C} / \mathrm{T}$ & $\mathrm{C} / \mathrm{T}$ & $\mathrm{EBP}(2)$ \\
\hline 6 & $36 / \mathrm{M}$ & $\mathrm{No}^{*}$ & $\mathrm{No}^{*}$ & $\mathrm{No}^{*}$ & Cons. tx \\
\hline 7 & $40 / F$ & $\mathrm{~T}$ & $\mathrm{~T}$ & $\mathrm{~T}$ & EBP (1) \\
\hline 8 & $28 / F$ & $\mathrm{~T}$ & $\mathrm{~T}$ & $\mathrm{~T}$ & $\operatorname{EBP}(3)$ \\
\hline 9 & $26 / F$ & $\mathrm{~T} / \mathrm{L}$ & $\mathrm{T} / \mathrm{L}$ & $\mathrm{T}$ & $\operatorname{EBP}(2)$ \\
\hline 10 & $17 / M$ & $\mathrm{~L}$ & $\mathrm{~L}$ & $\mathrm{~L}$ & Cons. tx \\
\hline 11 & $33 / F$ & $\mathrm{~T}$ & $\mathrm{~T} / \mathrm{L}$ & $\mathrm{C} / \mathrm{T}$ & EBP (2) \\
\hline 12 & $44 / F$ & $\mathrm{C} / \mathrm{T}$ & $\mathrm{C} / \mathrm{T}$ & $\mathrm{T}$ & $\mathrm{EBP}(1)$ \\
\hline 13 & $42 / F$ & $\mathrm{~L}$ & L & $\mathrm{C} / \mathrm{L}$ & EBP (1) \\
\hline 14 & $32 / F$ & $\mathrm{~L}$ & $\mathrm{~L}$ & $\mathrm{~T} / \mathrm{L}$ & EBP (1) \\
\hline 15 & 40/M & $\mathrm{T}$ & $\mathrm{T}$ & $\mathrm{C} / \mathrm{T}$ & $\operatorname{EBP}(2)$ \\
\hline
\end{tabular}

Note: $-C$ indicates cervical spine; $T$, thoracic spine; L, lumbar spine; cons. TX, conservative treatment; EBP, epidural blood patch.

* CSF leakage was not detected on MRM or RIC.

peated EBP. In 6 patients, follow-up MRM was performed between 14 days and 6 months after EBP placement. In all 6 patients, high signal intensities around the nerve root sleeves disappeared or showed much improvement, suggesting amelioration of CSF leak on follow-up MRM (Fig 3).

\begin{tabular}{|c|c|c|c|}
\hline & \multicolumn{2}{|c|}{ MRM } & \multirow[t]{2}{*}{ RIC } \\
\hline & Reader 1 & Reader 2 & \\
\hline $\begin{array}{r}\text { Sensitivity } \\
95 \% \mathrm{Cl}\end{array}$ & $\begin{array}{c}0.867 \\
(0.694-1.000)\end{array}$ & $\begin{array}{c}0.800 \\
(0.598-1.000)\end{array}$ & $\begin{array}{c}0.933 \\
(0.807-1.000\end{array}$ \\
\hline $\begin{array}{r}\text { Specificity } \\
95 \% \mathrm{Cl}\end{array}$ & $\begin{array}{c}0.867 \\
(0.694-1.000)\end{array}$ & $\begin{array}{c}0.933 \\
(0.807-1.000)\end{array}$ & - \\
\hline $\begin{array}{l}\text { Accuracy } \\
95 \% \mathrm{Cl}\end{array}$ & $\begin{array}{c}0.867 \\
(0.745-1.000)\end{array}$ & $\begin{array}{c}0.867 \\
(0.745-1.000)\end{array}$ & - \\
\hline
\end{tabular}

Note:- $95 \% \mathrm{Cl}$ indicates $95 \%$ confidence interval; -, not available.

\section{Discussion}

We performed RIC and MRM on 15 patients with spinal CSF leak syndrome to detect the leakage site in the spine. CSF leak was detected in 14 patients by RIC and in 12-13 by MRM, depending on the different readers. In the control group, there were 1-2 subjects with false-positive findings. In our study, clinical criteria were used for the diagnosis of the disease. The sensitivity, specificity, and accuracy of MRM for detecting CSF leak were $86.7 \%, 86.7 \%$, and $86.7 \%$ in reader 1 , respectively, and $80.0 \%, 93.3 \%$, and $86.7 \%$ in reader 2 , respectively.

Spinal CSF leak syndrome is rare, causing postural headache and low CSF pressure due to CSF leak into the extradural space with no known history of iatrogenic dural injury. ${ }^{7}$ The pathogenesis of spinal CSF leak syndrome is considered to be 

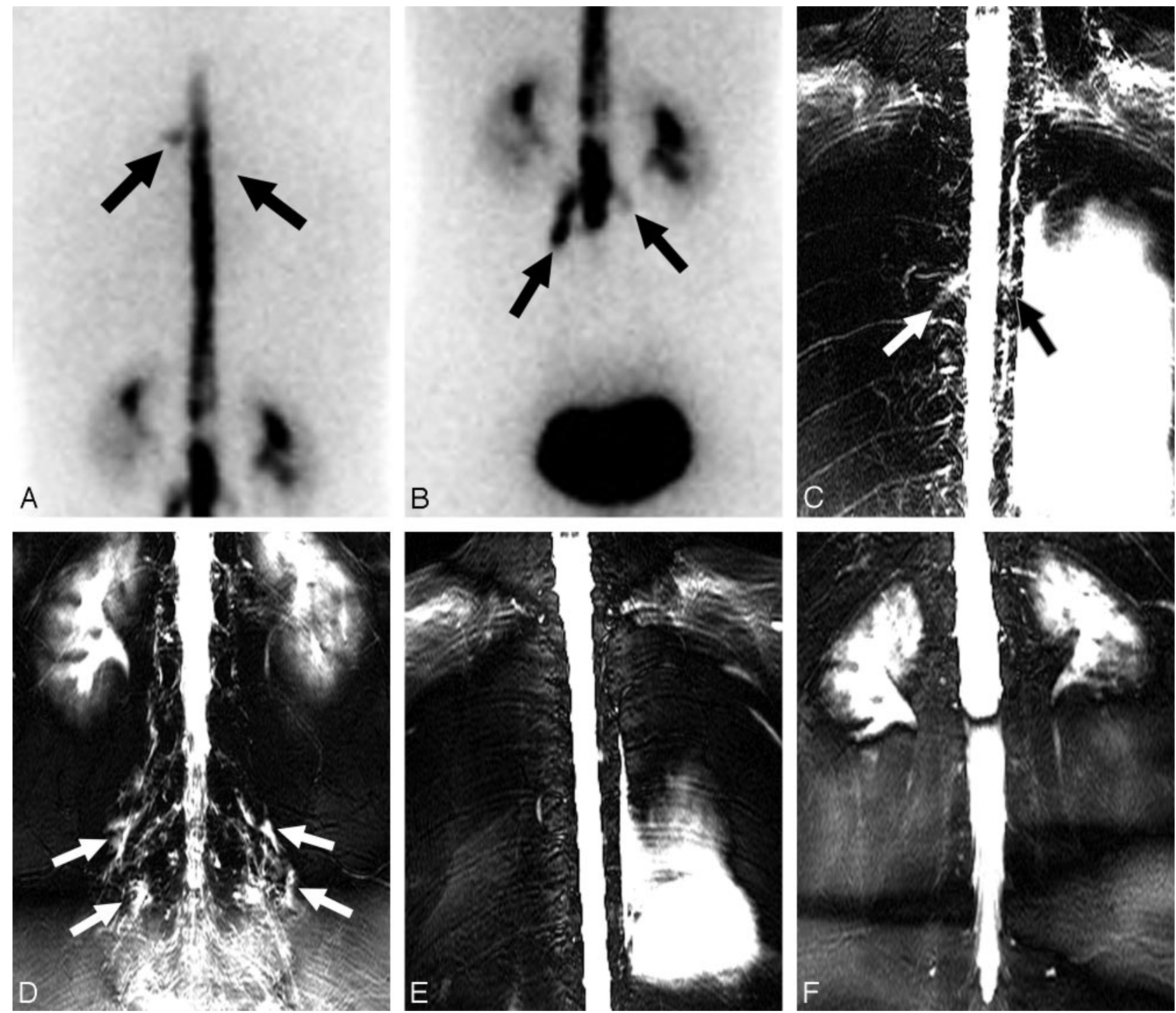

Fig 3. A 33-year-old woman with spinal CSF leak syndrome and multiple CSF leaks in the bilateral thoracic and lumbar spine. $A$ and $B$, Posterior projection of RIC shows diffusion of the radioisotope into the extra-arachnoidal space in the region of the upper thoracic spine (arrows), predominantly on the left side $(A)$ and in the lumbar spine $(B)$. $C$ and $D, M R M$ shows hyperintensities along multiple nerve root sleeves in the upper thoracic spine (arrows, C) and in the lumbar spine (arrows, D). Eand F, On 1-month follow-up MRM, multiple hyperintensities around the nerve root sleeves disappeared.

related to a CSF leak through small dural defects, resulting in decreased CSF volume and low CSF pressure. ${ }^{7,8}$ Although a spontaneous leak from an arachnoid diverticulum has been described in the literature, a structural lesion is seldom identified. ${ }^{9,10}$

Spinal CSF leak syndrome is generally considered to be benign, and most patients improve with conservative management. However, symptoms persist in some patients, and occlusion of the CSF leak is therefore required for symptomatic control. EBP is considered the treatment of choice for such patients. ${ }^{4,11}$ The mechanism of symptom relief by EBP has not yet been established. There are 2 hypotheses for effective pain relief. One proposes that the blood volume injected by EBP leads to increasing pressure in the subarachnoid space. ${ }^{5}$ The other hypothesis proposes that a rapid coagulation response stops CSF leak. ${ }^{12}$ Although some authors reported that the location of injection in EBP is not important, ${ }^{11}$ others suggested that injection should be targeted at the leakage site on the basis of the hypothesis that a blood clot may stop the CSF leak. ${ }^{13,14}$ We agree that, if at all possible, blood should be injected at the site of leakage. For this reason, we consider identification of the leakage site important for the successful treatment of spinal CSF leak syndrome.

Various diagnostic techniques, such as MR imaging, MRM, CTC, and RIC, may be used to detect the CSF leakage site. Spinal MR imaging in spinal CSF leak syndrome usually reveals extradural fluid collections, spinal meningeal enhancement, and dilation of the epidural venous plexus. ${ }^{15,16}$ In most cases, the search for the point of CSF leak is difficult and unsuccessful by using spinal MR imaging. ${ }^{17}$ CTC can provide evidence of meningeal anatomic defects, the precise location of extradural collections, and the relationship of the leakage site to bony structures. ${ }^{15}$ However, it is usually difficult to detect the exact leakage site, and screening of the whole spine may require a large amount of radiation exposure.

RIC may directly reflect the CSF dynamics. CSF leak can be 
directly visualized if there is an accumulation of radioactivity outside the subarachnoid space. The radiotracer may ascend slowly over the convexities or may quickly disappear from the subarachnoid space and then accumulate in the bladder. ${ }^{18}$ If there is no active leakage or if the size of the leakage is smaller than the resolution, no direct evidence of the leak is seen. ${ }^{19,20}$ RIC offers a more panoramic view of the entire spine, even though the sensitivity of RIC is not very high. ${ }^{21}$ Recent studies have reported the improved sensitivity of RIC in detecting CSF leak. ${ }^{22}$ In our series, the sensitivity of RIC appeared quite high compared with that in the previous reports. This improved detection rate in our study may be due to our accumulated experience with spinal CSF leak syndrome. The inherent drawback of RIC includes its invasiveness and possible radioisotope extravasation through the needle tract. Inaccuracy caused by postpuncture radioisotope leakage seems, however, to be negligible.

Various MRM techniques have been used to evaluate disk disease, traumatic pseudomeningocele, or other diseases of the spine. The basic principle of MRM is to enhance the CSF signal intensity by suppressing the adjacent tissue signal intensity. MRM eliminates the signal intensity from epidural fatty tissue by using an extremely long TE and effective fat suppression..$^{23,24}$

A few sporadic cases have been reported of the detection of a CSF leak site in spinal CSF leak syndrome by using MRM. $^{25-27}$ Matsmura et al ${ }^{26}$ reported a stripe of CSF leak along a nerve root. We did not, however, encounter a similar finding in our 15 cases. Yoshimoto et $\mathrm{al}^{27}$ and Chiapparini et $\mathrm{al}^{15}$ reported patients with CSF leak that appeared similar to our cases. In the study by Yoshimoto et al, the spinal canal had fully expanded to the nerve root sleeves and high-intensity streaks were observed along with extraspinal nerve bundles on MRM. In the study by Chiapparini et al, a long and irregular root sleeve was also observed on MRM.

We used the 2D TSE technique for MRM. Earlier in our study, we tried various techniques including the balanced fastfield echo and 2D and 3D TSE. In our experience, 2D TSE visualized the leakage site better than other techniques. In addition, the acquisition time of the 2D TSE sequence is very short and took only 72 seconds for each level and 144 seconds to cover the entire spine. A detailed discussion of the MRM technique is, however, beyond the scope of our study.

On MRM, CSF leak appeared as CSF space expansion around nerve root sleeves, with various degrees of diffuse, streaky, and irregular high signal intensity along the nerve root sleeves and extraspinal area. The underlying mechanism of the diffuse high signal intensities along the nerve root sleeves on MRM in our study is not clear. We speculate that they may reflect the fluid collection itself or a secondary phenomenon of CSF leak such as engorgement of the venous or lymphatic system for drainage of excessive fluid. These streaky high signal intensities disappeared on follow-up studies in patients with improved symptoms. Our results concerning the leakage pattern and multiple-level involvement of the spine may suggest clues for the explanation of the mechanism of CSF leak in spinal CSF leak syndrome. Sometimes, perineurial cysts or meningocele may mimic CSF leak on MRM. However, in contrast to CSF leak, there is no triangular-shaped expansion of the nerve root sleeves or associated streaky hyperintensities along the nerve root (Fig 2C).

The most common sites of CSF leak in spinal CSF leak syndrome have been reported as the cervicothoracic junction and the thoracic area. ${ }^{28}$ Our study showed similar results. Multiple simultaneous spinal CSF leaks in patients on their first presentation are well described in the literature. ${ }^{18,21,27,29,30}$

There are limitations to our study. The most important one is that we used clinical criteria as the reference standard to evaluate the leakage. However, in considering the lack of ultimate diagnostic tools for spinal CSF leak syndrome, we believe it is reasonable to use the clinical criteria. Another limitation is the variability in identifying leakage sites between readers and between the MRM and RIC techniques. The variability had 2 causes. One was the difficulty in identifying the exact level of the leak on MRM as well as RIC. For example, in cases of leak at the cervicothoracic junction level, there was some discrepancy of level identification between the readers and between the study techniques. Another cause was that there were locations of subtle changes in addition to the more obvious lesions in each study. This is partly due to our limited experience in this disease. In addition, our grading system is arbitrary and is not fully tested yet. We used the grading system only to determine the presence of the CSF leak and did not directly compare the results of grading between the 2 readers of the MRM or between MRM and RIC. The grading system we proposed needs further critical evaluation.

\section{Conclusion}

The diagnostic performance of MRM for spinal CSF leak syndrome is comparable to that of RIC, and MRM is an effective tool for the evaluation of CSF leak in spinal CSF leak syndrome. MRM can be used as a screening diagnostic technique. We believe that MRM should be tried before RIC or CTC; and if the findings of MRM are positive, clinical treatment with EBP is reasonable. This would reserve the other techniques for equivocal cases or cases with negative MRM findings and impressive clinical findings.

\section{Acknowledgment}

We thank Bonnie Hami, MA, Department of Radiology, University Hospitals Health System, Cleveland, Ohio, for editorial assistance in preparing the manuscript.

\section{References}

1. Chung SJ, Kim JS, Lee MC. Syndrome of cerebral spinal fluid hypovolemia: clinical and imaging features and outcome. Neurology 2000;55:1321-27

2. Mokri B, Atkinson JL, Piepgras DG. Absent headache despite CSF volume depletion (intracranial hypotension). Neurology 2000;55:1722-24

3. Mokri B, Hunter SF, Atkinson JL, et al. Orthostatic headaches caused by CSF leak but with normal CSF pressures. Neurology 1998;51:786-90

4. Sencakova D, Mokri B, McClelland RL. The efficacy of epidural blood patch in spontaneous CSF leaks. Neurology 2001;57:1921-23

5. Carrie LE. Epidural blood patch: why the rapid response? Anesth Analg 1991;72:129-30

6. Chung SJ, Ahn JH, Lee JH, et al. Orthostatic hypacusis in a patient with CSF hypovolaemia. Cephalalgia 2006;26:613-16

7. Rando TA, Fishman RA. Spontaneous intracranial hypotension: report of two cases and review of the literature. Neurology 1992;42:481-87

8. Pannullo SC, Reich JB, Krol G, et al. MRI changes in intracranial hypotension. Neurology 1993;43:919-26

9. Mokri B. Spontaneous intracranial hypotension. Curr Neurol Neurosci Rep 2001;1:109-17 
10. Schievink WI, Meyer FB, Atkinson JL, et al. Spontaneous spinal cerebrospinal fluid leaks and intracranial hypotension. J Neurosurg 1996;84:598-605

11. Berroir S, Loisel B, Ducros A, et al. Early epidural blood patch in spontaneous intracranial hypotension. Neurology 2004;63:1950-51

12. DiGiovanni AJ, Galbert MW, Wahle WM. Epidural injection of autologous blood for postlumbar-puncture headache. II. Additional clinical experiences and laboratory investigation. Anesth Analg 1972;51:226-32

13. Hayek SM, Fattouh M, Dews T, et al. Successful treatment of spontaneous cerebrospinal fluid leak headache with fluoroscopically guided epidural blood patch: a report of four cases. Pain Med 2003;4:373-78

14. Kantor D, Silberstein SD. Cervical epidural blood patch for low CSF pressure headaches. Neurology 2005;65:1138

15. Chiapparini L, Farina L, D'Incerti L, et al. Spinal radiological findings in nine patients with spontaneous intracranial hypotension. Neuroradiology 2002;44: 143-50, discussion 151-52

16. Clarot F, Callonnec F, Douvrin F, et al. Giant cervical epidural veins after lumbar puncture in a case of intracranial hypotension. AJNR Am J Neuroradiol 2000;21:787-89

17. Pleasure SJ, Abosch A, Friedman J, et al. Spontaneous intracranial hypotension resulting in stupor caused by diencephalic compression. Neurology 1998;50:1854-57

18. Benamor M, Tainturier C, Graveleau P, et al. Radionuclide cisternography in spontaneous intracranial hypotension. Clin Nucl Med 1998;23:150-51

19. Weber WE, Heidendal GA, de Krom MC. Primary intracranial hypotension and abnormal radionuclide cisternography: report of a case and review of the literature. Clin Neurol Neurosurg 1991;93:55-60

20. Molins A, Alvarez J, Sumalla J, et al. Cisternographic pattern of spontaneous liquoral hypotension. Cephalalgia 1990;10:59-65
21. Jeon TJ, Lee JD, Lee BI, et al. Radionuclide cisternography in spontaneous intracranial hypotension with simultaneous leaks at the cervicothoracic and lumbar levels. Clin Nucl Med 2001;26:114-16

22. Horikoshi $\mathrm{T}$, Ikegawa $\mathrm{H}$, Uchida $\mathrm{M}$, et al. Tracer clearance in radionuclide cisternography in patients with spontaneous intracranial hypotension. Cephalalgia 2006;26:1010-15

23. El Gammal T, Sobol W, Wadlington VR, et al. Cerebrospinal fluid fistula: detection with MR cisternography. AJNR Am J Neuroradiol 1998;19:627-31

24. Rabin BM, Roychowdhury S, Meyer JR, et al. Spontaneous intracranial hypotension: spinal MR findings. AJNR Am J Neuroradiol 1998;19:1034-39

25. Katramados A, Patel SC, Mitsias PD. Non-invasive magnetic resonance myelography in spontaneous intracranial hypotension. Cephalalgia 2006;26:1160-64

26. Matsumura A, Anno I, Nose T, et al. Intracranial hypotension. J Neurosurg 2001;95:914-16

27. Yoshimoto K, Takeshita I, Inoue T, et al. Multi-level disruption of the spinal nerve root sleeves in spontaneous spinal cerebrospinal fluid leakage: two case reports. Neurol Med Chir (Tokyo) 2001;41:154-59

28. Ali SA, Cesani F, Zuckermann JA, et al. Spinal-cerebrospinal fluid leak demonstrated by radiopharmaceutical cisternography. Clin $\mathrm{Nucl} \mathrm{Med}$ 1998;23:152-55

29. Arai M, Takada T, Ichijo K. A case of spontaneous intracranial hypotension with simultaneous CSF leaks at the upper and lower thoracic levels [in Japanese]. Rinsho Shinkeigaku 2001;41:775-79

30. Schievink WI, Jacques L. Recurrent spontaneous spinal cerebrospinal fluid leak associated with "nude nerve root" syndrome: case report. Neurosurgery 53:1216-18, 2003, discussion 1218-19 